\title{
V2 Trigeminal Referred Pain from Chronic Occipital Neuralgia Caused by Entrapment of the Greater Occipital Nerve
}

\author{
Chang-ik Lee ${ }^{1}$ Byung-chul Son ${ }^{1,2}$ \\ ${ }^{1}$ Department of Neurosurgery, Seoul St. Mary's Hospital, Seoul, \\ Republic of Korea \\ ${ }^{2}$ College of Medicine, Catholic Neuroscience Institute, The Catholic \\ University of Korea, Seoul, Republic of Korea
}

\begin{abstract}
Address for correspondence Byung-chul Son, MD, PhD, Department of Neurosurgery, Seoul St. Mary's Hospital, Catholic Neuroscience Institute, College of Medicine, The Catholic University of Korea, 222 Banpo-daero, Seocho-gu, Seoul, Republic of Korea 06591, (e-mail: sbc@catholic.ac.kr).
\end{abstract}

\begin{abstract}
Keywords

- decompression

- greater occipital nerve

- lacrimation

- occipital neuralgia

- trigeminocervical complex

Although entrapment of the greater occipital nerve (GON) is a well-known cause of occipital neuralgia, occurrence of referred hemifacial trigeminal pain involving V2 distribution from chronic occipital neuralgia is rare. A 67-year-old female patient with intermittent left-sided occipital neuralgia of 10 -year duration presented with a new onset of left-sided hemifacial pain of 5-month duration. With aggravation of left-sided occipital neuralgia, continuous burning pain and paresthesia gradually developed in her left malar and periorbital area. They also spread to her left upper lip. Severe compression of the left GON by tendinous aponeurotic attachment of the trapezius was found intraoperatively. Decompression of the left GON from chronic entrapment resulted in immediate relief for her hemifacial pain and chronic occipital neuralgia. These findings provide clinical affirmation of the existence of trigeminal/cervical convergence and hypersensitivity. Chronic irritating afferent input of occipital neuralgia caused by entrapment of the GON seems to be associated with sensitization and hypersensitivity of the second-order neurons in the trigeminocervical complex receiving convergent input from dural and cervical structures. Referred trigeminal pain from chronic occipital neuralgia may extend to V2 in addition to V1 trigeminal distribution.
\end{abstract}

\section{Introduction}

Occipital neuralgia is defined as a paroxysmal, shooting, or stabbing pain in the posterior part of the scalp, in the distribution(s) of the greater, lesser, and/or third occipital nerves. ${ }^{1-3}$ Although it has been suggested that the pain of occipital neuralgia can reach the fronto-orbital (V1) area through trigeminocervical interneuronal connections in the trigeminal spinal nucleus, ${ }^{1}$ its referral to V2 (mandibular) division is extremely rare.

Occurrence of hemifacial sensory changes (hypesthesia and paresthesia) associated with referred hemifacial pain involving all three trigeminal distributions from chronic occipital neuralgia caused by pathologic vascular contact of the greater occipital nerve (GON) has been reported recently. ${ }^{4}$

received

August 3, 2018

accepted

August 31, 2018

published online

March 28, 2019
Here we report a case of referred hemifacial pain mainly involving V2 trigeminal distribution from chronic occipital neuralgia in a 67-year-old female patient. Her occipital neuralgia was found to be caused by entrapment of the GON at its piercing point of the tendinous aponeurotic attachment of the trapezius at the superior nuchal line.

\section{Case Report}

A 67-year-old female patient with a 10-year history of intermittent stabbing pain in her left retroauricular suboccipital pain presented with a left-sided facial pain of 5-month duration. An intermittent, dysesthetic aching pain and mild tenderness over the left occipital area were associated with initial development of left occipital pain
DOI https://doi.org/

$10.1055 / \mathrm{s}-0038-1676662$ ISSN 2277-954X.
(C2019 Neurological Surgeons'

Society of India
License terms

() (1) $\ominus \circledast$ 
10 years ago. It occurred two or three times a year and lasted for about 3 weeks on every occasion. The severity of her left occipital pain was mild to moderate (3 5 out of 10 on numerical rating scale NRS-11, ranging from 0 to 10 ). It was controlled with nonsteroidal anti-inflammatory drugs (NSAIDs) and gabapentin (600 mg/day). It then subsided gradually without specific interventional treatment.

Five months prior to presentation, ill-defined, continuous burning pain and tingling sensation developed insidiously in her left malar and periorbital area, which gradually extended to the left upper lip (-Fig. 1). Within 2 weeks, repeated attacks of aching pain developed in her left cheek, temple, and occipital area. The onset was described to be rather sudden. Pain occurred two or three times a day and lasted for about 3 hours. The patient visited an emergency department. However, no abnormal finding was evident in computed tomography (CT) or magnetic resonance angiography (MRA). Under the impression of trigeminal neuralgia, the patient was treated with several NSAIDs, including carbamazepine $(600 \mathrm{mg} / \mathrm{day})$, tramadol (150 mg/day), and gabapentin (900 mg/day), with some degree of relief. Occipital nerve block over the tender point along the course of the GON was effective for the initial three injections. However, its effect lasted only 3 hours thereafter. Two percent lidocaine was not effective for block of supra- and infraorbital nerves. Considering the chronic nature of her unexplained left facial and occipital pain, the patient was referred for further evaluation of trigeminal and occipital pain.
Upon examination, her left suboccipital pain was found to be that of occipital neuralgia fulfilling the criteria of International Classification of Headache Disorders (ICHD). ${ }^{1}$ Her stabbing occipital pain was present throughout the day with intermittent aggravation in the distribution of the left GON ( - Fig. 1A). The tenderness was mild in examination. Her hemifacial pain was the most severe in her left malar area, periorbital, and temple ( - Fig. 1B). It was burning and aching in nature. There was no objective sensory abnormality such as hypoesthesia or hyperesthesia, allodynia, or tenderness in her left hemiface. Cranial nerve examinations were normal. There was no neck pain, neck tenderness, or limitation of neck motion. Her medical history was unremarkable, including diabetes. There were no symptoms or signs of trigeminal autonomic cephalalgia, including photophobia, nausea, vomiting, or conjunctival injection. Interestingly, the patient had subjective feeling of malar swelling and lacrimation during attack of left hemifacial pain. No specific dental cause or paranasal sinusitis was identified.

Laboratory examinations were normal, including erythrocyte sedimentation rate and C-reactive protein, antinuclear antibody, and anti-DsDNA. No pathologic lesion to explain her left facial and occipital pain was found in enhanced magnetic resonance imaging (MRI) of the brain or cervical spine. Additional treatment with triptan or ergotamine was not effective. Considering chronicity and referred nature of her hemifacial pain associated with preexisting occipital neuralgia, exploration of the left GON was proposed.
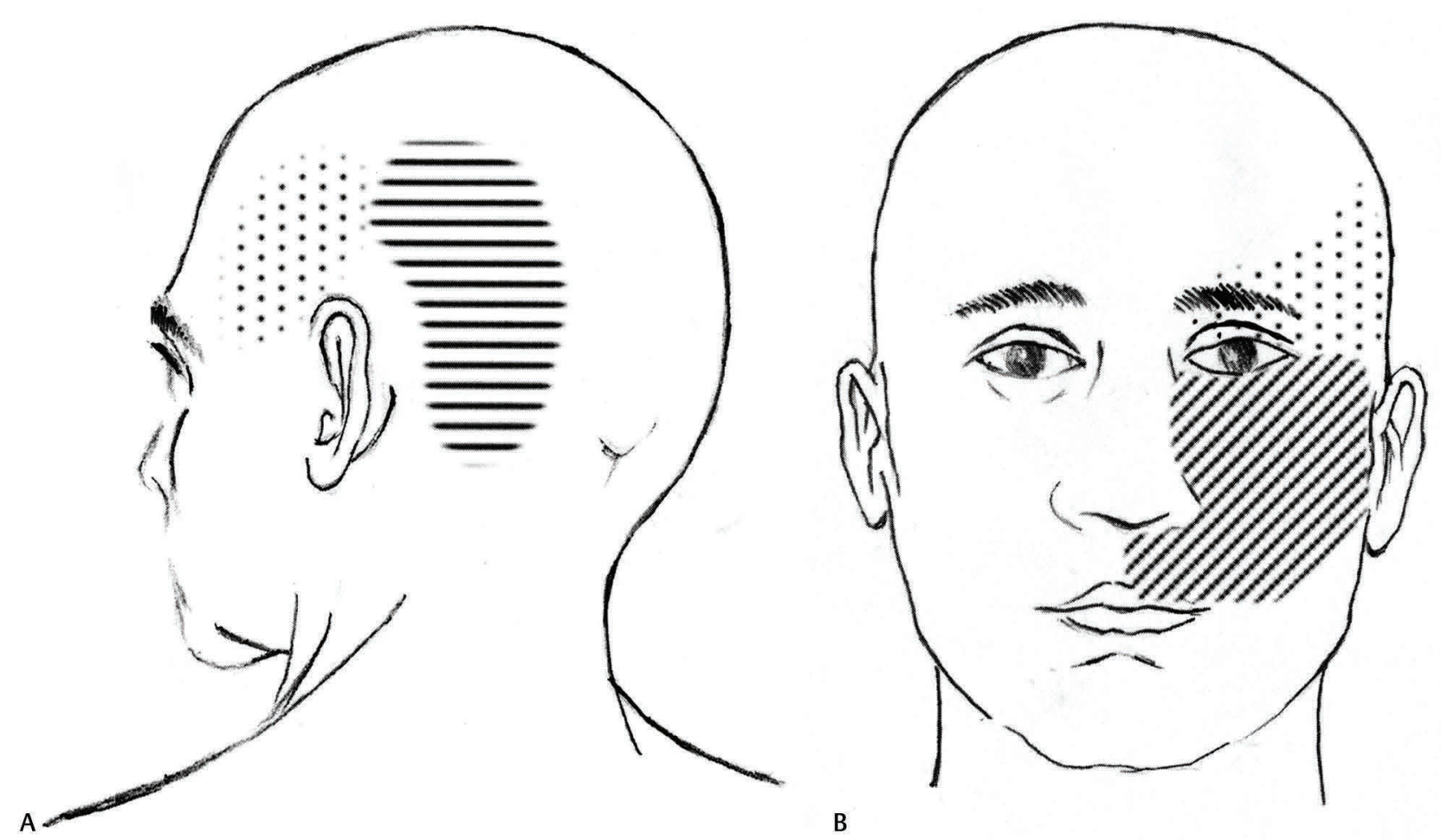

Fig. 1 Diagrams demonstrating the distribution of occipital neuralgia and hemifacial pain. (A) Horizontally hatched area over the left retroauricular occipital area indicates the distribution of stabbing pain of occipital neuralgia. Spotted area in the left temple denotes regions of aching pain. (B) Obliquely hatched area over the left hemiface indicates the distribution of burning and aching pain in the distribution of left V1 and V2 dermatomes. It was more severe in the left periorbital and malar area. III-defined sensation of malar congestion and ipsilateral lacrimation were associated with each pain attack. 
A hockey stick-shaped incision the size of $8 \mathrm{~cm}$ along the course of left GON was made. An oblique incision was then developed over the trapezius fascia. The left GON was identified within the semispinalis capitis muscle. After proximal dissection of semispinalis capitis and inferior obliquus capitis muscles from the GON, it was then carried distally, releasing the GON within the trapezial tunnel (the site where the GON penetrated through the trapezial aponeurotic attachment to the occiput). The GON was found to be severely entrapped by the aponeurotic arch of the trapezius, and it showed an indentation for chronic entrapment (-Fig. 2A). After releasing the trapezial aponeurotic arch, surrounding occipital artery and vein abutting the GON were dissected free (-Fig. 2B).

Immediately after decompression of the left GON, chronic stabbing pain of the occipital neuralgia and hemifacial pain at left periorbital, temple, and malar area including
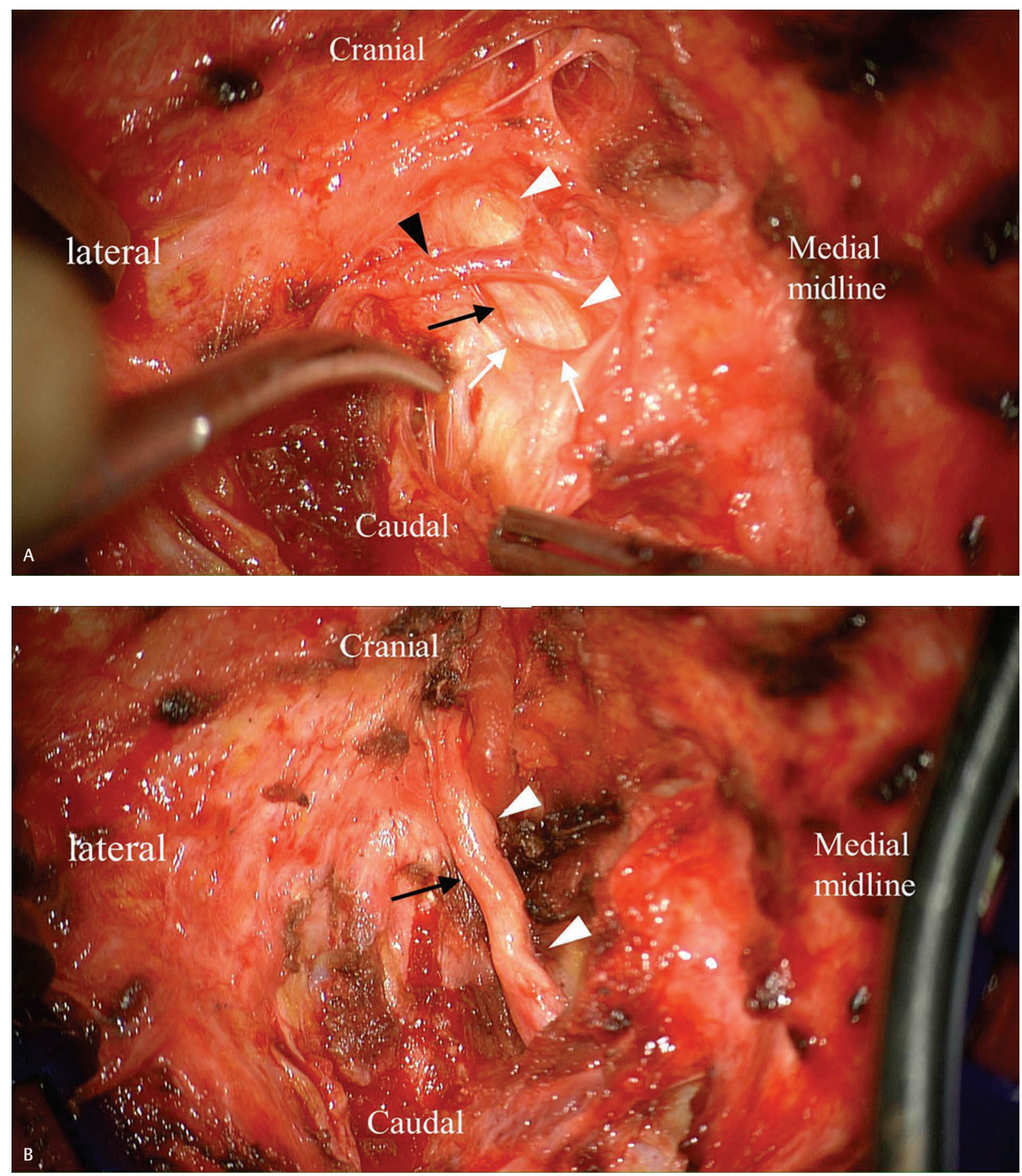

Fig. 2 Intraoperative photographs during decompression of the left greater occipital nerve (GON). (A) An intraoperative photograph showing severe entrapment of the GON (white arrowheads) by the aponeurotic fibrous attachment of the trapezius (white arrows) to the superior nuchal line. The maximum compression point is indicated by black arrow. Note the occipital artery (black arrowhead) is located superficially, abutting to the GON. (B) An intraoperative photograph after decompression of the left GON. The GON (white arrowheads) was decompressed with division of trapezial aponeurosis. Its proximal and distal courses are addressed. Note prompt restoration of thickness of the GON at the maximal constriction point (black arrow). 
left upper lip and dysesthetic burning sensation completely disappeared. Unpleasant sensation of malar congestion and unilateral lacrimation associated with attack of left hemifacial pain that occurred during the past 5-month aggravation also disappeared completely. No sensory deficit in the distribution of the GON or hemiface was observed. Mild dysesthesia with foreign-body sensation in the territory of the GON distal to the scalp incision site developed. It was controlled with gabapentin (600 mg/day) for 2 months. Her dysesthesia disappeared at the third postoperative month. Her left-sided occipital pain and left hemifacial pain did not recur at follow-up of 12 months.

\section{Discussion}

\section{Occipital Neuralgia due to Nerve Compression/Entrap- ment of the Nerve}

Occipital neuralgia is defined as unilateral or bilateral paroxysmal, stabbing, or shooting pain in posterior region of the scalp, or in areas where the greater and lesser occipital nerves are distributed..$^{1-3}$ Although the cause of occipital neuralgia is mostly unknown, it might be related to various vascular, muscular, neurogenic, and osteogenic lesions involving C2 dorsal root, extradural C2 root, and the greater and lesser occipital nerves..$^{5-8}$ The GON is originated from medial branch of dorsal rami of the second cervical nerve root. ${ }^{7.9}$ It also receives a branch from dorsal rami of $\mathrm{C} 3$ nerve. It ascends through the semispinalis muscle and then runs rostrolaterally before emerging into the scalp by piercing the aponeurotic fibrous attachment of the trapezius to the superior nuchal line. ${ }^{5-9}$ This aperture (trapezial tunnel) is a common site of entrapment of the GON. ${ }^{7-9}$ In this case, chronic occipital neuralgia was found to be caused by entrapment of the GON at the trapezial tunnel. Decompression of the GON immediately relieved the referred hemifacial pain and chronic occipital neuralgia.

\section{Referred Hemifacial Pain from Chronic Occipital Neuralgia}

Occurrence of referred hemifacial pain from occipital neuralgia is extremely rare. However, stimulation of structures in the neck innervated by upper cervical roots in humans such as infratentorial dura mater, vessels, and tumors of the posterior fossa can lead to pain in the frontal head. ${ }^{10,11}$ Furthermore, cluster headache, a rare form of primary headache marked by unilateral excruciating pain with autonomic features, was associated with ipsilateral vertebral artery aneurysm ${ }^{12}$ and vertebral artery dissection. ${ }^{13}$ Secondary cluster headache from a vertebral artery aneurysm could be resolved when the aneurysm was clipped ${ }^{12}$ or treated with sumatriptan injection..$^{13}$ Occurrence of hemifacial hypesthesia and paresthesia associated with referred hemifacial pain of trigeminal distribution from chronic occipital neuralgia due to pathological vascular contact of the GON has been reported recently. ${ }^{4}$ Another case of referred hemifacial pain to ipsilateral V1 and V2 trigeminal distribution from chronic occipital neuralgia is reported here. These findings provide clinical affirmation of the existence of trigeminal/cervical convergence and hypersensitivity.
Trigeminocervical complex (TCC) is a population of neurons in the $\mathrm{C} 2$ dorsal horn that receive convergent input from facial skin corresponding to the trigeminal nerve and cervical structures innervated by high cervical C1-3 roots. ${ }^{10,11,14,15}$ Convergence of nociceptive afferents and sensitization of TCC neurons have clinical correlates, including hypersensitivity and the spread and referral of pain frequently seen in patients with primary headaches such as migraines. ${ }^{10,11,14}$ Patients with primary headaches often report pain involving not only the frontal head innervated by the trigeminal nerve but also the occipital region innervated by the GON. ${ }^{11}$ Sensitization of central nociceptive neurons in the TCC will occur in response to strong dural noxious inputs seen in secondary headache syndromes such as meningitis, subarachnoid hemorrhage, and experimental headaches. ${ }^{14}$ Chronic compression and adhesion of the GON by the occipital artery ${ }^{4,8}$ and its constriction by the aponeurotic fibrous aperture of the trapezius muscle can cause chronic occipital neuralgia. ${ }^{5-7}$ Chronic noxious afferent input of occipital neuralgia may lead to central sensitization of the TCC and result in referred hemifacial trigeminal pain. ${ }^{4}$

\section{Conclusion}

A rare occurrence of referred hemifacial pain of trigeminal distribution from chronic occipital neuralgia is reported here. Chronic entrapment of the GON at its course along tendinous aponeurotic attachment of the trapezius caused occipital neuralgia. Unremitting, chronically increased peripheral afferent stimuli of occipital neuralgia resulted in sensitization of central nociceptive neurons in TCC, producing ipsilateral V2 hemifacial trigeminal pain.

\section{Financial Support and Sponsorship \\ None.}

\section{Conflicts of Interests}

The authors declare no conflict of interest regarding this manuscript and that this manuscript has not been previously published in whole or in part or submitted elsewhere for review.

\section{Institutional Review Board Approval}

All procedures performed in this study were in accordance with the ethical standards of the institutional and/or national research committee and with the 1964 Helsinki declaration and its later amendments or comparable ethical standards. This study was approved by our university's institutional review board.

\section{References}

1 Headache Classification Committee of the International Headache Society (IHS). The International Classification of Headache Disorders. 3rd ed. Cephalalgia 2018;38:1-211

2 Son BC, Kim DR, Lee SW. Intractable occipital neuralgia caused by an entrapment in the semispinalis capitis. J Korean Neurosurg Soc 2013;54(3):268-271

3 Vanelderen P, Lataster A, Levy R, Mekhail N, van Kleef M, Van ZundertJ.8. Occipital neuralgia. Pain Pract 2010;10(2):137-144 
4 Son BC, Choi JG. Hemifacial pain and hemisensory disturbance referred from occipital neuralgia caused by pathological vascular contact of the greater occipital nerve. Case Rep Neurol Med 2017;2017:3827369

5 Mosser SW, Guyuron B, Janis JE, Rohrich RJ. The anatomy of the greater occipital nerve: implications for the etiology of migraine headaches. Plast Reconstr Surg 2004;113(2):693697, discussion 698-700

6 Ducic I, Moriarty M, Al-Attar A. Anatomical variations of the occipital nerves: implications for the treatment of chronic headaches. Plast Reconstr Surg 2009;123(3):859-863, discussion 864

7 Cesmebasi A, Muhleman MA, Hulsberg P, et al. Occipital neuralgia: anatomic considerations. Clin Anat 2015;28(1):101-108

8 Cornely C, Fischer M, Ingianni G, Isenmann S. Greater occipital nerve neuralgia caused by pathological arterial contact: treatment by surgical decompression. Headache 2011;51(4):609-612

9 Standring S, Borley NR, Collin P, et al. Gray's Anatomy. Philadelphia, PA: Elsevier; 2008
10 Bartsch T, Goadsby PJ. Stimulation of the greater occipital nerve induces increased central excitability of dural afferent input. Brain 2002;125(Pt 7):1496-1509

11 Piovesan EJ, Kowacs PA, Tatsui CE, Lange MC, Ribas LC, Werneck LC. Referred pain after painful stimulation of the greater occipital nerve in humans: evidence of convergence of cervical afferences on trigeminal nuclei. Cephalalgia 2001;21(2):107-109

12 West P, Todman D. Chronic cluster headache associated with a vertebral artery aneurysm. Headache 1991;31(4):210-212

13 Cremer PD, Halmagyi GM, Goadsby PJ. Secondary cluster headache responsive to sumatriptan. J Neurol Neurosurg Psychiatry 1995;59(6):633-634

14 Bartsch T, Goadsby PJ. The trigeminocervical complex and migraine: current concepts and synthesis. Curr Pain Headache Rep 2003;7(5):371-376

15 Goadsby PJ, Knight YE, Hoskin KL. Stimulation of the greater occipital nerve increases metabolic activity in the trigeminal nucleus caudalis and cervical dorsal horn of the cat. Pain 1997;73(1):23-28 